\title{
Alfabloqueantes en pacientes con hiperplasia benigna de próstata complicada por vejiga hiperactiva: ¿son todos igualmente eficaces? Estudio SNIPER
}

\section{Alphablockers in patients with benign prostatic hyperplasia complicated by overactive bladder: Are they all equally effective? SNIPER study}

Antonio Alcántara Montero
Centro Salud José María Álvarez, Don Benito, Badajoz España.

Correspondencia: Dr. Antonio Alcántara Montero a.alcantara.montero@hotmail.com
Aproximadamente 50-75\% de los pacientes con hiperplasia benigna de próstata con síntomas de la vía urinaria baja tienen síntomas de vejiga hiperactiva. ${ }^{1}$ Los alfa-1 bloqueantes se prescriben para tratar a los pacientes con hiperplasia benigna de próstata con vejiga hiperactiva, porque no solo disminuyen los síntomas de vaciado, sino también los de Ilenado, incluidos los de vejiga hiperactiva.

Los receptores $\alpha 1$ adrenérgicos se encuentran en la vejiga, cuello vesical y próstata. Se han identificado tres subtipos de receptores adrenérgicos: $\alpha 1 \mathrm{~A}, \alpha 1 \mathrm{~B}$ y $\alpha 1 \mathrm{D}$. En la vejiga, el subtipo $\alpha 1$ más frecuente es el $\alpha 1 \mathrm{D}$, en el cuello vesical existen el $\alpha 1 \mathrm{D}$ y el $\alpha 1 \mathrm{~A}$, mientras que en la próstata predomina el subtipo $\alpha 1 \mathrm{~A}$ en $98 \%{ }^{2}$

Pero, ¿son todos los alfa-1 bloqueantes igualmente eficaces en pacientes con hiperplasia benigna de próstata y vejiga hiperactiva? Algunos estudios han examinado el efecto de silodosina en comparación con otros alfa- 1 bloqueantes en pacientes con síntomas de la vía urinaria bajahiperplasia benigna de próstata. ${ }^{3,4}$ Sin embargo, no se habían publicado hasta la fecha estudios a gran escala, con asignación al azar, con alfa- 1 bloqueantes que comparasen la disminución de los síntomas de vejiga hiperactiva en pacientes con hiperplasia benigna de próstata y vejiga hiperactiva, de acuerdo con la diferencia de selectividad por el receptor adrenérgico $\alpha 1 \mathrm{~A}$.

Naftopidil, un alfa-1 bloqueante con mayor selectividad para el subtipo de receptor adrenérgico $\alpha 1 \mathrm{D}$ que para el subtipo $\alpha 1 \mathrm{~A}$, es más eficaz en la disminución de los síntomas de llenado que tamsulosina (que tiene mayor selectividad para el receptor adrenérgico $\alpha 1 \mathrm{~A}$ que para el $\alpha 1 \mathrm{D}$ )..$^{5-7}$ La silodosina es el alfa-1 bloqueante más urose- 
lectivo en los receptores $\alpha 1 \mathrm{~A}$ que ha demostrado disminuir los síntomas de vejiga hiperactiva en pacientes con síntomas en la vía urinaria baja con hiperplasia benigna de próstata. ${ }^{2}$

El estudio SNIPER, recientemente publicado en The Journal of Urology, es el primero que compara la eficacia de dos alfa-1 bloqueantes con diferente afinidad para los subtipos de receptores adrenérgicos $\alpha 1$, silodosina y naftopidil, en el tratamiento de la hiperplasia benigna de próstata complicada por vejiga hiperactiva. Es un estudio prospectivo, abierto, con asignación al azar, multicéntrico, de 12 semanas de duración, en 350 pacientes ambulatorios con hiperplasia benigna de próstata no tratada, con síntomas asociados de urgencia miccional de al menos una vez por semana y puntuación en el cuestionario de síntomas de vejiga hiperactiva (OABSS- overactive bladder symptom score) $\geq$ 3. Los pacientes se asignaron al azar para recibir $8 \mathrm{mg}$ al día de silodosina o $75 \mathrm{mg}$ al día de naftopidil. Los cambios en los parámetros desde el inicio hasta las 4 y 12 semanas se evaluaron según el IPSS (puntuación internacional de los síntomas prostáticos), IPSS-QOL (parámetro de calidad de vida del IPSS), OABSS, y las funciones de vaciado según la flujometría. ${ }^{8}$

Resultados: en el análisis de eficacia se incluyeron, en ambos grupos, 314 pacientes. La media del IPSS, IPSS-QOL, y OABSS mejoró significativamente en ambos grupos. Se observó una mejoría estadísticamente significativa mayor en el grupo silodosina que en el de naftopidil en el OABSS total ( $p=0.03)$, IPSS-QOL $(p=0.005)$, y en la puntuación de urgencia del OABSS $(p<$ 0.001 ) a las 12 semanas. En la función de vaciado, el flujo urinario máximo mostró mejoras significativas en ambos grupos, pero el cambio en la tasa de flujo máximo fue significativamente mayor en el grupo de silodosina $(3.6 \mathrm{~mL} / \mathrm{s})$ en comparación con la del grupo naftopidil (2.1 $\mathrm{mL} / \mathrm{s})$ a las 12 semanas. ${ }^{8}$
Seguridad: en cuanto a los efectos adversos, 7 pacientes (4\%) del grupo de silodosina y $8(4.6 \%)$ del grupo de naftopidil interrumpieron el tratamiento debido a efectos adversos. No se observó diferencia significativa entre los dos grupos, y ninguno de los pacientes experimentó retención urinaria. Además, las tasas de interrupción del tratamiento debido efectos adversos en este estudio fueron casi similares a las reportadas en estudios previos. Quince pacientes (9.6\%) en el grupo de silodosina y 5 (3.2\%) en el de naftopidil se quejaron de disfunción eyaculatoria. Si bien la incidencia de trastornos de la eyaculación fue mayor en el grupo de silodosina en comparación con la del grupo de naftopidil, ningún paciente suspendió el tratamiento en ninguno de los grupos. ${ }^{8}$

En conclusión, éste es el primer estudio que compara 2 alfa- 1 bloqueantes con diferentes perfiles de selectividad en los subtipos de receptores adrenérgicos; la silodosina (un alfa-1 bloqueante altamente selectivo sobre el subtipo de receptores adrenérgicos $\alpha 1 \mathrm{~A}$ ) demostró ventajas en la disminución de los síntomas de vejiga hiperactiva y flujo urinario en comparación con naftopidil (un alfa-1 bloqueante más selectivo en el subtipo de receptores adrenérgicos $\alpha 1 \mathrm{D})$. Esa mejora, quizá, se relaciona con la mayor selectividad del alfa-1 bloqueante por el receptor adrenérgico $\alpha 1 \mathrm{~A}$ y, por tanto, silodosina podría ser un tratamiento inicial de elección razonable para los pacientes con hiperplasia benigna de próstata y vejiga hiperactiva.

\section{REFERENCIAS}

1. Yamaguchi O, Nishizawa O, Takeda M, Yokoyama O, Homma $\mathrm{Y}$, Kakizaki $\mathrm{H}$, et al. Clinical guidelines for overactive bladder. Int J Urol. 2009;16:126-42.

2. Alcántara Montero A. Papel de los alfabloqueantes en el tratamiento de los síntomas del tracto urinario inferior/ hiperplasia benigna de próstata: ¿son todos iguales? Silodosina en la "vida real". Semergen. 2016;42(Supl 2):1-9. 
3. Shirakawa T, Haraguchi T, Shigemura K, Morishita S, Minayoshi K, Miyazaki J, et al. Silodosin versus naftopidil in Japanese patients with lower urinary tract symptoms associated with benignprostatic hyperplasia: a randomized multicenter study. Int J Urol. 2013;20:903-10.

4. Yamaguchi K, Aoki Y, Yoshikawa T, Hachiya T, Saito T, Takahashi S. Silodosin versus naftopidil for the treatment of benign prostatic hyperplasia: a multicenter randomized trial. Int J Urol. 2013;20:1234-8.

5. Nishino Y, Masue T, Miwa K, Takahashi Y, Ishihara S, Deguchi T. Comparison of two alpha1- adrenoceptor antagonists, naftopidil and tamsulosin hydrochloride, in the treatmentof lower urinary tract symptoms with benign prostatic hyperplasia: a randomized crossover study. BJU Int. 2006 ;97:747-51.
6. Ukimura O, Kanazawa M, Fujihara A, Kamoi K, Okihara $\mathrm{K}$, Miki T, et al. Naftopidil versus tamsulosin hydrochloride for lower urinary tract symptoms associated with benign prostatichyperplasia with special reference to the storage symptom: a prospective randomized controlled study. Int J Urol. 2008;15:1049-54.

7. Perumal C, Chowdhury PS, Ananthakrishnan N, Nayak $P$, Gurumurthy S. A comparison of the efficacy of naftopidil and tamsulosin hydrochloride in medical treatment of benignprostatic enlargement. Urol Ann. 2015;7:74-8.

8. Matsukawa Y, Funahashi Y, Takai S, Majima T, Ogawa T, Narita $\mathrm{H}$, et al. Comparison of silodosin and naftopidil for efficacy in the treatment of benign prostatic enlargement complicated by overactive bladder: A randomized, prospective study (SNIPER study). J Urol. 2016. doi: 10.1016/j.juro.2016.08.111. 